records were used to collect patient information and relevant laboratory values. Data was analysed for those in whom 1 year follow-up information was available.

Results 218 patients in NI (123 male, 95 female) underwent LT between 1st January 2010 and 31st December 2019. Age ranged from 13 to 71 at time of transplant (mean 44 years old). Complete records are available for 204 patients allowing at least one year follow-up. 13 patients were transplanted as super-urgent fulminant cases, with the rest transplanted on the elective list. 7 patients had known pre-existing CKD. 32 patients had hypertension and 43 were diabetic pre-transplant. Post-transplant, 101 patients were hypertensive, and 72 were known to be diabetic. At 1 year, 1 patient had CKD 5,10/204 had CKD and 110/204 patients had CKD 3. 94/204 (46\%) had a deterioration by at least 1 CKD stage. Of the 93 patients in whom 5 year data is available, 1 patient had CKD 5 (1.1\%), 2 had CKD 4 (2.2\%), and 48 had CKD 3 (51.6\%). 37 patients $(20.4 \%)$ are known to renal services. Based on NI GAIN guidelines, a further 22 patients (15.3\%) meet criteria for routine referral to nephrology based on sustained reduction of $>25 \%$ eGFR in one year based on recent blood results.

Conclusion Within LT recipients in Northern Ireland, the incidence of ESRD is much lower than quoted in the historical literature $(1.1 \%$ of current patients with CKD 5 and $2.2 \%$ CKD 4). A substantial proportion have CKD 3 (51.6\%). 61\% of patients have risk factors in addition to calcineurin inhibitors. Care of these patients should include regular monitoring of renal function as well as aggressive management of risk factors such as diabetes mellitus and hypertension.

\section{P013 RETROSPECT ANALYSIS OF PATIENTS WITH PRIMARY BILIARY CHOLANGITIS (PBC) IN A DISTRICT GENERAL HOSPITAL}

Yun Jie Chew, Savanthi Fernando*, John Hutchinson. Mid York NHS Trust, Wakefield, UK

\subsection{6/gutjnl-2021-BASL.22}

Introduction We performed a retrospective audit of the demographics, diagnoses and metabolic risk factors among patients with Primary Biliary Cholangitis (PBC) at Mid Yorkshire Hospitals NHS Trust (MYHT); MYHT provides hospital services to a population of 530,000 .

Methods Retrospective analysis of patient records and clinic letters was undertaken. We included patients who are currently having follow up with the Hepatology and or Gastroenterology services and reviewed their medical records for the past 3 years from December 2017 to December 2020.

Results 39 patients diagnosed with PBC are currently under the follow up of the local Hepatology outpatient department. $95 \%(n=37)$ are female. The median age is 62.5 years (range from 37 to 88 years old) with a median age of diagnosis of 55.5 years (range from 32 to 79 years old). The median of Body Mass Index (BMI) was 30.5 (range from 19 to 42$)$ with $3 \%(n=1)$ have alcohol intake exceeding the national guideline of less than 14 units a week. 98\% $(\mathrm{n}=38)$ were Anti-mitochondrial Antibodies (AMA) positive and 23\% $(n=9)$ was Antinuclear Antibody (ANA) positive.

$44 \%(n=17)$ was diagnosed with additional autoimmune conditions, with hypothyroidism being the commonest diagnosis $41 \%(n=7)$. Other autoimmune conditions include lichen planus, Grave's disease, Crohn' disease, CREST syndrome, Sarcoidosis and Vitiligo. 18\% $(n=7)$ had additional liver conditions; the commonest being Non- Alcoholic Fatty Liver disease (NAFLD) $(43 \%, \mathrm{n}=3) ; 27 \% \quad(\mathrm{n}=10)$ had hypertension and $18 \%(n=7)$ had dyslipidaemia.

$13 \%(n=5)$ of the patients had liver biopsy; for the initial diagnoses of PBC. $80 \%(n=4)$ of them had their liver biopsy for diagnostic purpose although they were already AMA seropositive. Only 20\% (n=1) had liver biopsy to investigate the possibility of autoimmune hepatitis overlap.

Conclusions The number of patients with PBC at Wakefield was 7.4 in 100,000 which was less than the prevalence in the UK which was 35 in 100,000, with female predominance $;^{1}{ }^{2}$ this suggests that we have undiagnosed $\mathrm{PBC}$ patients in our MYHT population. Additionally, we found a high prevalence of NAFLD in this population which affirms the importance of good metabolic risk factor management in this patient group in addition to the traditional targeted PBC therapies such as Ursodeoxycholic acid and fibrates.

\begin{tabular}{|c|c|}
\hline & Number of patients $(n=39)$ \\
\hline \multicolumn{2}{|l|}{ Age } \\
\hline $0-65$ & 23 \\
\hline$>65$ & 16 \\
\hline \multicolumn{2}{|l|}{ Gender } \\
\hline Female & 37 \\
\hline Male & 2 \\
\hline \multicolumn{2}{|l|}{ Age at diagnosis } \\
\hline $0-65$ & 35 \\
\hline$>65$ & 3 \\
\hline \multicolumn{2}{|l|}{ BMI } \\
\hline $18.5-25$ & 13 \\
\hline $25-30$ & 14 \\
\hline$>30$ & 11 \\
\hline \multicolumn{2}{|l|}{ AMA serology } \\
\hline Positive & 38 \\
\hline Negative & 1 \\
\hline \multicolumn{2}{|l|}{ ANA serology } \\
\hline Positive & 9 \\
\hline Negative & 28 \\
\hline Unknown & 2 \\
\hline \multicolumn{2}{|l|}{ Associated autoimmune condition } \\
\hline Hypothyroidism & 7 \\
\hline Lichen Planus & 2 \\
\hline Others & 8 \\
\hline \multicolumn{2}{|l|}{ Other liver diseases } \\
\hline Non- alcoholic fatty liver disease & 3 \\
\hline Others & 3 \\
\hline \multicolumn{2}{|l|}{ Co-morbidities } \\
\hline Hypertension & 10 \\
\hline Hypercholesterolemia & 7 \\
\hline
\end{tabular}




\section{REFERENCES}

1. Mid Yorkshire Hospitals NHS Trust. Trust Strategy 2017/2021. Available from: https://www.midyorks.nhs.uk/download/doc/docm93jijm4n4980.pdf?ver=6031

2. Hirschfield GM, Dyson JK, Alexander GJM, et al. The British society of gastroenterology/UK-PBC primary biliary cholangitis treatment and management guidelines. Gut 2018;67:1568-1594.

\section{P014 TREATMENT OF PRIMARY BILIARY CHOLANGITIS (PBC): RETROSPECTIVE REVIEW OF CURRENT THERAPIES IN A DISTRICT GENERAL HOSPITAL}

Yun Jie Chew*, Savanthi Fernando, John Hutchinson. Mid York NHS Trust, UK

\subsection{6/gutjnl-2021-BASL.23}

Introduction We retrospectively reviewed current therapies of patients with Primary Biliary Cholangitis (PBC) at Mid Yorkshire Hospitals NHS Trust (MYHT). MYHT provides hospital services to a population of 530,000 .

Methods We undertook a retrospective review of 542 patient records and clinic letters. Information was obtained from electronic patient records and Sunquest ICE system. We included patients who were under outpatient review in both the Hepatology and Gastroenterology services; over a 3 year period from December 2017 to December 2020.

Results $87 \%(n=34)$ of the patient were on ursodeoxycholic acid (UDCA) therapy. Among the patients who were not on UDCA therapy, $75 \%(n=3)$ was due to their liver function test being normal and therefore they were undergoing annual monitoring and $25 \% \quad(n=1)$ were due to medication side effects (e.g. diarrhoea).

Of the patients on UDCA therapy, 56\% $(\mathrm{n}=19)$ were on a therapeutic dose $(13-15 \mathrm{mg} / \mathrm{kg} / \mathrm{day}) .79 \%(\mathrm{n}=27)$ had achieved a good clinical response; biochemical normalisation in liver function tests (LFTs) with UDCA therapy with the remainder having a suboptimal biochemical response. No patient had been started on Obeticholic acid (OCA); however, 50\% $(n=4)$ of patients who have failed or intolerant to UDCA therapy were being considered for OCA therapy. Our audit showed that all patients with diagnosed PBC and abnormal LFTs had been offered and started on UDCA therapy. No patients were on fibrate therapy.

Discussions The standard recommended by the British Society of Gastroenterologists (BSG) is that $90 \%$ of patients receive a therapeutic dose of UDCA and documented if tolerant. Therefore, there is still room for improvement in our current practice. It is also essential to ensure diagnosed patients be started on UDCA therapy earlier and to be referred early for OCA therapy if they fail UDCA therapy. Bezafibrate or fibrate therapy should be considered as add-on therapy for

\begin{tabular}{ll} 
Abstract P014 Table 1 & \\
\hline & Number of patients $(\mathrm{n}=\mathbf{3 9})$ \\
\hline UDCA therapy & 34 \\
Ongoing & 5 \\
$\quad$ Not on treatment & \\
Dosage of UDCA therapy & 19 \\
$\quad$ Therapeutic & 15 \\
Subtherapeutic & \\
\hline
\end{tabular}

patients with incomplete response or intolerance to UDCA therapy. ${ }^{1} 2$

\section{REFERENCES}

1. Corpechot C. The role of fibrates in primary biliary cholangitis. Curr Hepatology Rep 2019;18:107-114. https://doi.org/10.1007/s11901-019-00455-3

2. Corpechot C, Chazouilleres O, Rousseau A, Le Gruyer A, Habersetzer F, Mathurin $\mathrm{P}$, et al. A placebo-controlled trial of bezafibrate in primary biliary cholangitis. $N$ Engl J Med 2018;378:2171-81.

\section{P015 RETROSPECTIVE ANALYSIS OF FOLLOW UP IN PRIMARY BILIARY CHOLANGITIS (PBC) IN A DISTRICT GENERAL HOSPITAL}

Yun Jie Chew*, John Hutchinson. Mid York NHS Trust, UK

\subsection{6/gutjnl-2021-BASL.24}

Introduction We performed a retrospective audit on improvement needed for follow up among patients with Primary Biliary Cholangitis (PBC) at Mid Yorkshire Hospitals NHS Trust (MYHT) from the perspective of symptom, treatment and follow up in comparison with the recommendation from British Society of Gastroenterology.

Methods Retrospective analysis of patient records and clinic letters. We included patients who are currently having follow up with the Hepatology and or Gastroenterology services and reviewed their medical records for the past 3 years. We will be summarising the improvement needed in follow up to achieve the standards recommended by the British Society of Gastroenterology.

Results With regards to the symptom management, 28\% $(n=11)$ complained of itching, but only $4 \% \quad(n=2)$ of the $28 \%$ have been prescribed first-line agents such as cholestyramine and $28 \%(n=11)$ of them has complained of fatigue as a symptom. This was usually attributed to being multifactorial but there was no structured approach in quantifying fatigue and its impact.

In terms of therapy, there was clear documentation on whether Ursodeoxycholic Acid has been started and the reason if it was not or it has been stopped. However, only 56\% $(n=19)$ were on the therapeutic dose.

$46 \%(n=18)$ have not had an up to date bone density scan (DEXA scan) and the reason was not documented in the clinic letter.

The standards recommended by the British Society of Gastroenterology (BSG) involve 90\% have the presence/absence of symptoms recorded in the notes and have suggested tools to quantify symptom such as tiredness. ${ }^{1}$ It is also recommended that $90 \%$ of patients receiving therapy at adequate dose. $^{1}$

Discussions All the patient with diagnosed PBC and deranged liver function tests had been offered UDCA therapy and clear documentation when they were not. Improvement is still needed to ensure that patient is on the therapeutic dose of UDCA therapy. Documentation of symptom needed in each review to assess the impact of the disease on quality of life using multi-domain PBC-40 QOL measure1. To achieve the goals set out by BSG, we have designed and implemented a clinical proforma to be used during clinical follow up with patients. We intend to perform a re-audit in a year time to ensure improvement in care for patients with PBC. 